\title{
ESTRATEGIAS INSTRUCCIONALES APLICADAS POR LOS DOCENTES PARA DESARROLLAR PROCESOS METACOGNITIVOS EN LOS ESTUDIANTES
}

\author{
INSTRUCTIONAL STRATEGIES APPLIED BY TEACHERS IN DEVELOPING STUDENTS \\ METACOGNITIVE PROCESSES
}

\author{
Meredith Jiménez-Cárdenas ${ }^{1}$ (D), Sileny Cujía-Berrio² ${ }^{\text {ID }}$ y Aquiles Mejía-Anaya ${ }^{3}$ (D)
}

\begin{abstract}
RESUMEN
Se analizaron las estrategias instruccionales aplicadas por docentes, en el desarrollo de procesos metacognitivos a estudiantes del Instituto Técnico Nacional de Formación Técnica Profesional (INFOTEP). Tipo de investigación analítica, con diseño no experimental transversal de campo. Representado por 67 docentes que laboran en el INFOTEP del Municipio de San Juan del Cesar, Guajira - Colombia, y 830 estudiantes del período II-2014. Se aplicó la técnica de la encuesta y observación participante se realizó instrumento con 30 ítems y cinco alternativas de respuestas cerradas, La validez mediante juicio de expertos, y confiabilidad del coeficiente de Alpha Cronbach, alcanzando un valor de 0,85 . Se concluyó que existen diferencias en las respuestas entre docentes y estudiantes, con relación a las estrategias instruccionales y la percepción del proceso metacognitivo de los alumnos; se observó un nivel de correspondencia moderado entre ambas variables. Las estrategias aplicadas por docentes no han permitido consolidar de manera plena los conocimientos que contribuyan al manejo efectivo de procesos cognitivos por parte de los estudiantes y, por consiguiente, a alcanzar un aprendizaje significativo. Se recomienda diseñar jornadas de reflexión para socializar los resultados, a fin de delinear acciones que contribuyan al desarrollo de los procesos metacognitivos, en relación al conocimiento, a su base cognitiva, que puedan conducir a un aprendizaje significativo.
\end{abstract}

Palabras clave: Estrategias instruccionales; procesos metacognictivos; autorregulación.

\section{ABSTRACT}

This research focused on instructional strategies applied by teachers at the National Institute of Professional Technical Training (INFOTEP) to help students develop metacognitive processes. It was an analytical research, with non-experimental cross-field design. The corpus consisted of 67 teachers working at the INFOTEP in San Juan del Cesar, Guajira-Colombia, as well of 830 of their students during the second semester of 2014. The technique applied was the survey and the instrument, which

\footnotetext{
Fecha de recepción: enero 01 de 2016 / Fecha de aceptación: marzo 9 de 2017 / Publicado en línea: abril 19 de 2017 Tipología: Artículo de Investigación Científica y Tecnológica

Para citar este artículo: Jiménez, C. M., Cujía, B. S. \& Mejía, A. A. (2017). Estrategias instruccionales aplicadas por los docentes para desarrollar procesos metacognitivos en los estudiantes. Praxis, 13(1), 25 - 36. Doi: http://dx.doi.org/10.21676/23897856.2064
}

\footnotetext{
1. Universidad de la Guajira, Magíster en Educación con Énfasis en Cognición. Doctor en Ciencias Mención Gerencia (URBE - Venezuela), Correo electrónico: meredith@uniguajira.edu.co ORCID: 0000-0002-7894-420X

2. Instituto Nacional de Formación Técnica Profesional INFOTEP, Especialista en Recreación Ecológica y Social. Doctor en Ciencias Mención Gerencia (URBE

- Venezuela), Correo electrónico: scujia@infotep.edu.co ORCID: 0000-0002-1262-9776

3. Universidad Rafael Belloso Chacín. Magíster en Gerencia de Proyectos Investigación y Desarrollo, Doctor en Ciencias Mención Gerencia (URBE - Venezuela), Correo electrónico: amejaya@gmail.com ORCID: 0000-0002-3968-234X
} 
was participant observation, contained 30 items and 5 choices of closed answers. The validity by expert judgment, and the reliability of the coefficient of Alpha Cronbach, reached a value of 0.85 . The conclusion of the study is that teachers and students differ in their perception of instructional strategies and of the metacognitive process of the students. A moderate level of correspondence was observed between both variables. Strategies applied by teachers have not helped to fully consolidate knowledge that contributes to the students effective management of cognitive processes and consequent meaningful learning. It is recommended that discussion sessions be scheduled to socialize the results, in order to design actions that contribute to mend this situation.

Keywords: Instructional Strategies; Metacognitive processes; self-regulation.

\section{INTRODUCCIÓN}

$\mathrm{E}$ n un mundo posmoderno la educación es una vía para la búsqueda de nuevas alternativas que orienten las transformaciones sociales. Lo cual exige la producción de innovaciones radicales que permitirán acrecentar la eficacia de los sistemas de aprendizaje para vencer los obstáculos que impiden al participante el desarrollo de su creatividad, y poder asimilar la transferencia de conocimientos entre instructor-educando, de tal forma que logre mejorar las habilidades y destrezas en situaciones de la vida real.

A nivel mundial la educación percibe cambios trascendentales en el área de las comunicaciones, la tecnología, lo científico, lo ideológico que influyen en los diferentes grupos sociales que sostienen el progreso y la calidad de vida de los habitantes. El Conjunto de entidades educativas, específicamente las de formaciones técnicas, tiene que amoldarse a las cambiantes exigencias actuales para garantizar las carencias de esas transformaciones sociales.

Uno de los avances tecnológicos que se dan en el proceso de aprendizaje dispuesto en la educación básica y superior es un nuevo modelo que proyecta la educación aplicando estrategias divergentes, permitiendo explorar la capacidad de aprender y pensar. Se infiere, por consiguiente, la necesidad de plantear la transformación del proceso de aprendizaje en el nivel superior porque las universidades deberán hacer frente a los retos que suponen las nuevas tecnologías.
Es importante resaltar en esta parte que el éxito de las estrategias instruccionales (actividades, ejercicios, problemas o cualquier tipo de experiencia lúdicopedagógica) que aplica el docente, radica en las técnicas y métodos; a tal efecto deberá dejar de lado las viejas prácticas tradicionales, ya que este proceso exige que haya más interacción entre el docente y el estudiante, que escuche activamente sus necesidades, brinde retroalimentación continua, fomente su creatividad y, sobre todo, ser empático. Visto desde esta perspectiva, el acompañamiento está llamado a fortalecer la autonomía personal en la interacción constructiva y a movilizar el pensamiento creativo de los estudiantes.

De esa forma, se detalla que en el contexto colombiano, existe la necesidad de estrategias instruccionales aplicadas por los docentes en el marco del desarrollo de los procesos metacognitivos de los estudiantes. En este sentido Valencia, Gil, Betancourt, Giraldo \& Orozco (2011) afirman que en algunas instituciones existen estudiantes que se distraen con frecuencia, se dispersan de la clase, y en general no prestan atención. De allí , la necesidad de que el docente utilice estrategias, instrumentos y técnicas para lograr la atención, facilitar la integración en el aula de clase.

Por tanto, es importante conocer si los docentes cuentan con los conocimientos necesarios en relación a las estrategias instruccionales. Por su parte Maussa (2014) manifiesta que la evaluación debe ser constante y se requiere juzgar el desempeño del pedagogo, ya que constituye un 
acuerdo con los procesos administrativos de institución educativa. Dichas estrategias se pueden utilizar antes, durante y al cierre de la sesión de aprendizaje, o si se encuentran en constante actualización de sus conocimientos a los fines de mejorar la praxis educativa.

En ese contexto el Instituto Nacional de Formación Técnica Profesional (INFOTEP) de San Juan del Cesar no es ajeno a esta problemática; ha observado a una muestra del personal docente, supervisor y estudiantes, a través de la experiencia directa de la investigadora y la aplicación de entrevistas diagnósticas no estructuradas, vivenciando que algunos profesores del INFOTEP utilizan muchas metodologías tradicionales para el desarrollo de las clases con ausencia de creatividad, innovación, espacios tanto de motivación como de mediación, e incluso muestran y transmiten desinterés en los temas desarrollados y en ocasiones se presentan casos de improvisación de las mismas.

Parte de esta problemática se presenta dentro del INFOTEP donde, según observaciones informales realizadas por la investigadora, se denotó que la mayoría de los estudiantes se encuentran fuera de sus aulas durante su horario de clases porque no tienen suficiente motivación para permanecer en sus clases; por lo cual se asevera que una de las posibles causas de esta situación es la falta de preparación de contenidos por los docentes, el empleo de herramientas didácticas dentro del proceso de aprendizaje, así como la motivación necesaria en el uso de estrategias instruccionales es bajo. Es por esta razón que los estudiantes no obtienen un aprendizaje cognitivo más efectivo y eficaz, que les permita tener una investigación formativa y que, según Agudelo, Martínez \& Ortiz (2013), dinamice los vínculos reales y latentes entre el conocimiento y los procesos académicos.

Por otra parte, en reuniones formales y conversatorios, los docentes del INFOTEP expresan que sus alumnos se encuentran indiferentes durante las clases, poco interesados y no se evidencia en su rendimiento académico un incremento del aprendizaje en las distintas asignaturas. Expresan los docentes que sus métodos básicos para impartir las asignaturas son las clases magistrales y proyección de videos.

En el marco de esas ideas, el estudio estuvo orientado a analizar las estrategias instruccionales aplicadas por los docentes, en el marco del desarrollo de los procesos metacognitivos de los estudiantes del INFOTEP del Departamento de La Guajira - Colombia.

\section{METODOLOGÍA}

Este planteamiento está inmerso en el modelo epistemológico positivista con la finalidad de establecer una realidad sistémica de forma general y homogénea, logrando la ponderación detallada de la información, consiguiendo una enorme conexión, aptitud y certeza. En apoyo a lo planteado, Leal (2009) identifica la actitud creativa la cual implica pasión y autoestima; y que para ser creativo hay que estar dispuesto a romper moldes, a someterlos a la crítica, a equivocarse y a aprender hasta lograr los propósitos esperados.

En ese sentido, la investigación empleó técnicas cuantitativas. Efectuó cálculos sobre los niveles de respuestas suministradas por la muestra estudiada según las dimensiones establecidas, para medir el comportamiento de la variable en estudio y el criterio de análisis utilizado; se confrontó la teoría con los hechos a través de la observación, verificación y el análisis científico y de esta manera poder analizar las estrategias instruccionales correspondientes al conjunto de actividades, ejercicios, problemas, o cualquier tipo de experiencia que puedan aplicar los docentes en el marco del desarrollo de los procesos metacognitivos de los estudiantes del INFOTEP del Departamento de La Guajira - Colombia.

La investigación se ubicó en la tipología analítica y, de acuerdo con Hurtado (2000), contempla estas características como un sistema reflexivo, lógico, cognitivo que conlleva sacar pautas de relaciones internas de un evento, contextos, fenómenos, entre otras. De igual forma, la autora 
agrega este tipo de investigación de carácter analítico tiene como propósito profundizar en un evento y comprenderlo en términos de sus propiedades poco tangibles. Por otro lado, según Bunge (1981), la investigación analítica pretende exteriorizar los elementos que conforman la totalidad y las conexiones que descifran su integración.

Para efectos del presente estudio se utilizó un diseño no experimental, transaccional descriptivo (Hernández, Fernández \& Batista, 2010) y de campo, pues se recolectaron datos de forma directa aplicando un cuestionario a las fuentes de información primarias, representadas por los docentes y estudiantes.

De acuerdo con esta óptica para la presente investigación se consideraron dos poblaciones: la primera representada por el total de 67 docentes que laboran en el INFOTEP del Municipio de San Juan del Cesar, Departamento de La Guajira Colombia y, la segunda por los 830 estudiantes inscritos para el período II-2014 en cada uno de los programas. En el caso de los docentes se utilizó criterio de censo poblacional (Tamayo \& Tamayo, 2007), para los estudiantes se utilizó un muestreo probabilístico estratificado a través de la fórmula de Sierra-Bravo, por ser la estadística más apropiada para el tipo de estudió, quedando conformada por la muestra por 270 estudiantes; se aplicó la fórmula de Shiffer, citado por Chávez (2007), cuya fórmula es la siguiente:

$$
\mathbf{n} 1=\mathbf{n} \cdot \mathbf{h} \cdot \mathbf{n}
$$

Donde:

$\mathrm{n} 1$ = Estado a determinar, $\mathrm{n}=$ tamaño adecuado de la muestra, $\mathrm{h}=$ tamaño del estrato de la población., $\mathrm{N}=$ tamaño de la población.

Para la recolección de datos se diseñó sobre la base del cuadro de operacionalización de la variable un cuestionario cerrado estructurado en 30 íteme, con cinco alternativas de respuesta cerrada (Siempre, casi siempre, a veces, casi nunca y nunca) el cual fue validado a través de la aplicación del juicio de 7 expertos, y establecida su confiabilidad utilizando el coeficiente Alpha de Crombach. En tal sentido, por ser un cuestionario construido con una escala de actitudes cuya fórmula es la siguiente:

$$
r_{k k}=(k /(k-1))\left[1-\left(\sum S^{2} i / S^{2} t\right)\right]
$$

Donde:

$\mathrm{K}=\mathrm{N}^{0}$ de Item, $\mathrm{Si}=$ Varianza de cada ítems. St2 = Varianza de los Puntajes Totales. $\mathrm{X}=$ Media.

Según Vieytes "cabe confiar en un instrumento cuando produce constantemente los mismos resultados al aplicarlo a una misma muestra" (2004, p.445). Este coeficiente tiene la condición de calcular la Varianza de cada ítems y la de los puntajes totales así como la media aritmética, arrojando un valor de 0.85 , lo cual indica un alto nivel del cuestionario y su asertiva aplicación.

Para la interpretación de los datos se utilizó derivado del enfoque cuantitativo del estudio, el método de estadística descriptiva tomando como estadígrafos la media y la desviación estándar; la primera con el propósito de establecer la tendencia de las respuestas, y la segunda para determinar el nivel de confiabilidad de las mismas.

\section{RESULTADOS Y DISCUSIÓN}

Por otra parte, en relación a los resultados alcanzados por el procesamiento para los estudiantes, se observa una diferencia significativa en cuanto a su opinión con relación a las estrategias instruccionales que aplican los docentes al ubicarse el promedio en un nivel moderado $(3,04)$, con una muy baja dispersión de 0,42 (Tabla 1). 
Tabla 1. Variable: Estrategias Instruccionales

\begin{tabular}{|l|c|c|c|c|}
\hline \multirow{2}{*}{ Dimensiones } & \multicolumn{2}{c|}{ Docentes } & \multicolumn{2}{c|}{ Estudiantes } \\
\cline { 2 - 5 } & Promedio & Desviación & Promedio & Desviación \\
\hline Tipos de estrategias instruccionales & 4,00 & 0,31 & 3,04 & 0,42 \\
\hline $\begin{array}{l}\text { Métodos de formación de estrategias } \\
\text { instruccionales }\end{array}$ & 3,99 & 0,30 & 3,04 & 0,43 \\
\hline $\begin{array}{l}\text { Criterios para la aplicación de las } \\
\text { estrategias instruccionales }\end{array}$ & 3,97 & 0,27 & 3,04 & 0,42 \\
\hline Promedio de la variable & 3,99 & 0,29 & 3,04 & 0,42 \\
\hline
\end{tabular}

Fuente: Datos obtenidos mediante la aplicación del cuestionario a docentes y estudiantes de las instituciones analizadas (Jiménez, Cujía, Mejía,2015).

En ese marco de ideas, Tobón (2010) el profesor es el principal actor en la transformación de los sistemas educativos en todos sus niveles; las competencias para utilizar adecuadamente estrategias y técnicas instruccionales son un aspecto fundamental para el desarrollo de los procesos formativos coherentes, que conduzcan a alcanzar un aprendizaje significativo. En la práctica se requiere que los docentes conozcan y dominen diversas estrategias instruccionales o para el aprendizaje, además del uso eficiente de las telecomunicaciones y los recursos de información.

En cuanto a la primera dimensión en estudio, referida a los tipos de estrategias instruccionales, el procesamiento de los datos arrojó en el caso de los docentes un promedio de 4 y una desviación de 0,31 ; indica una categoría alta con una muy baja dispersión de las respuestas, al referir que con alta frecuencia aplican dentro de su ejercicio un conjunto de estrategias y recursos instruccionales que les permiten antes, durante y posterior al proceso de aprendizaje manejar de forma efectiva las condiciones que demarcan el hecho educativo como una manera de contribuir con el aprendizaje significativo de los estudiantes.

Con diferencias de opinión, los estudiantes perciben que solo de forma moderada son utilizadas estas estrategias por parte de los docentes al arrojar un promedio de 3,04 y la desviación 0,42; se indica una muy baja dispersión que refiere uniformidad en las respuestas de los alumnos, lo cual se traduce en una muy alta confiabilidad de las mismas.
Para Díaz \& Hernández (2002), los tipos de estrategias instruccionales representan el conjunto de actividades, ejercicios, problemas o cualquier tipo de experiencia que el docente utiliza para guiar el proceso de aprendizaje desde que inicia hasta que termina, de manera que facilita la consecución del objetivo. Exponen al respecto que las mismas deben estar en consonancia las características, intereses, necesidades, expectativas y motivación de los alumnos.

En ese sentido se detallan a continuación los resultados alcanzados para cada uno de los indicadores que permitieron la operacionalización de esta dimensión. Al respecto, en cuanto a las estrategias preinstruccionales, el procesamiento de los datos arrojó para los docentes un promedio de 4,01 y una desviación de 0,34, ubicándose en la categoría alta con una muy baja dispersión de las respuestas, al expresar que con alta frecuencia preparan al estudiante a la hora de iniciar una sesión de aprendizaje; activan sus conocimientos previos, evocan experiencia de manera que se conectan efectivamente con los nuevos conocimientos presentados, creando al mismo tiempo -antes de iniciar una sesión de aprendizaje- expectativas sobre la base de los objetivos que se pretenden alcanzar.

Los estudiantes, por el contrario, ubicaron sus respuestas en un nivel moderado al arrojar el promedio un valor de 3,03 y la desviación 0,42, indicando que con moderada frecuencia los docentes los preparan a la hora de iniciar una 
actividad de aprendizaje como manera para activar conocimientos previos, evocar experiencias y crear expectativas que contribuyan a elevar su motivación durante el proceso.

En cuanto a los estudiantes, el promedio alcanzado fue de 3,06 y la desviación de 0,44, indicando una categoría moderada con una muy baja dispersión de las respuestas, al indicar que durante el proceso instruccional con modera frecuencia los docentes utilizan las estrategias anteriormente mencionadas.
Con respecto al último indicador de esta dimensión, relacionada a las estrategias posinstruccionales, como se observa en la tabla 2 , el promedio alcanzado para los docentes fue de 3,99 y la desviación de 0,24 al indicar que con alta frecuencia utilizan estrategias que permiten al estudiante formarse una visión sintética e integradora del aprendizaje adquirido; aplican además estrategias que permiten al alumno valorar su propio aprendizaje, y favorecer en la práctica, la retención, de información relevante.

Tabla 2. Dimensión: Tipos de estrategias instruccionales

\begin{tabular}{|l|c|c|c|c|}
\hline \multirow{2}{*}{\multicolumn{1}{c|}{ Indicadores }} & \multicolumn{2}{c|}{ Docentes } & \multicolumn{2}{c|}{ Estudiantes } \\
\cline { 2 - 5 } & Promedio & Desviación & Promedio & Desviación \\
\hline Estrategias preinstruccionales & 4,01 & 0,34 & 3,03 & 0,42 \\
\hline Estrategias coinstruccionales & 4,01 & 0,24 & 3,06 & 0,44 \\
\hline Estrategias posinstruccionales & 3,99 & 0,35 & 3,04 & 0,40 \\
\hline Promedio de la variable & 4,00 & 0,31 & 3,04 & 0,42 \\
\hline
\end{tabular}

Fuente: Datos obtenidos mediante la aplicación del cuestionario a docentes y estudiantes de las instituciones analizadas (Jiménez, Cujía, Mejía, 2015).

Con relación a las respuestas de los estudiantes el promedio alcanzado fue de 3,04 y la desviación de 0,40, ubicándose en la categoría moderada con una muy baja dispersión de las respuestas al expresar que con moderada frecuencia los docentes aplican este tipo de estrategias al momento de culminar la sesión de aprendizaje.

De acuerdo con Díaz \& Hernández, las estrategias posinstruccionales permiten al estudiante formarse una visión sintética, integradora hasta reflexiva y crítica del aprendizaje adquirido. En este sentido "Se presentan después del contenido que se ha de aprender, y permiten al alumno formar una visión sintética, integradora incluso critica del material” (2002, p.230). En otros casos le permiten valorar su propio aprendizaje.

En tanto a la dimensión relacionada a los métodos de formación de estrategias instruccionales, el procesamiento arrojó en el caso de los docentes un promedio de 3,99 y una desviación de 0,30; indicó una categoría alta con una muy baja dispersión de las respuestas, al expresar los profesores que con alta frecuencia utilizan la información y el modelado como métodos para la formación de estrategias instruccionales durante el manejo de las sesiones de aprendizaje.

Por su parte, reflejando la tendencia de las respuestas en cuanto a las diferencias de percepción entre docentes y estudiantes, los alumnos consideran que solo de manera moderada los profesores utilizan este tipo de métodos al arrojar el procesamiento un promedio de 3,04 y una desviación de 0,43.

En ese aspecto señalado, para Díaz \& Hernández (2002), la formación del docente como un profesional debe abordar dos cuestiones importantes: "las características personales que los hacen eficaces y la delimitación de los métodos de enseñanza eficaces" (p.13). La primera preocupación que debe adoptarse al tratar estas 
cuestiones es la ausencia de un método ideal, válido para cualquier objetivo y para cualquier situación a nivel educativo ya sea en el aula o a nivel administrativo. Para los autores citados la concordancia entre el contenido de la formación y el método seleccionado para lograr una estrategia instruccional es un factor más en la eficacia del ejercicio docente, como base para alcanzar un aprendizaje significativo.

Comprender estos resultados exige profundizar los datos arrojados por el procesamiento para cada uno de los indicadores que operacionalizan esta dimensión. En este sentido, en cuanto al indicador información, el procesamiento arrojó para los docentes un promedio de 4 y una desviación de 0,27 indicando una categoría alta con una muy baja dispersión de las respuestas, al expresar los profesores que con alta frecuencia participan en actividades formativas que contribuye a optimar sus competencias para el manejo de los procesos de aprendizaje, se preocupan por buscar información que contribuya a mejorar su praxis educativa, buscando además participar activamente en programas de formación de manera de profundizar sus conocimientos en relación al manejo de estrategias instruccionales.

Por su parte, la percepción de los estudiantes, con relación al indicador información y a la participación de los docentes en actividades formativas, es de nivel moderado al arrojar el promedio un valor de 3,05 y la desviación 0,43 (Tabla 3).

Tabla 3. Dimensión: Métodos de formación de estrategias instruccionales

\begin{tabular}{|l|c|c|c|c|}
\hline \multirow{2}{*}{\multicolumn{1}{|c|}{ Indicadores }} & \multicolumn{2}{c|}{ Docentes } & \multicolumn{2}{c|}{ Estudiantes } \\
\cline { 2 - 5 } & Promedio & Desviación & Promedio & Desviación \\
\hline Información & 4,00 & 0,27 & 3,05 & 0,43 \\
\hline Modelado & 3,98 & 0,34 & 3,04 & 0,44 \\
\hline Promedio de la Variable: & 3,99 & 0,30 & 3,04 & 0,43 \\
\hline
\end{tabular}

Fuente: Datos obtenidos mediante la aplicación del cuestionario a docentes y estudiantes de las instituciones analizadas (Jiménez, Cujía, Mejía, 2015).

Para De la Hera (2004), en general, todos los tipos de información pueden proporcionarse a los docentes. Al mismo tiempo, el sentido y el propósito de tales mensajes en un contexto específico influirán en el impacto que tenga para el alumno y en la apropiación de determinados sistemas motivacionales en detrimento de otros. El autor refiere, en este sentido, que la información permite mejorar las estrategias instruccionales y es quizás el método más empleado.

Con relación al indicador modelado el procesamiento arrojó para los profesores un promedio de 3,98 y una desviación de 0,34, indicando un categoría alta y una muy baja dispersión de las respuestas al expresar que con una alta frecuencia presentan información a los estudiantes mediante un modelo que muestra las respuestas deseadas en una situación determinada; asumen el modelaje de sus supervisores como una fuente de mejoramiento continuo de su praxis académica, resaltando así la "enseñanza a través de la reflexión en la acción” (Schön, 1992, p.156). Evidencia la comunicación entre el educador y el estudiante es una situación básica para una enseñanza práctica reflexiva. Así mismo, el mentor transfiere mensajes a sus principiantes de manera verbal como en modo de ejecutar.

Por su parte, los estudiantes ubicaron sus respuestas en un nivel moderado al expresar que con esa frecuencia los docentes utilizan el modelado como método de formación de estrategias, pues como se observa el promedio fue de 3,04 y la desviación de 0,44.

Díaz \& Hernández, afirman que "el alumno al interactuar, construir y verificar los significados 
de lo que ve y oye, ejecuta las prescripciones del docente a través de la imitación reflexiva derivada del modelado del maestro" (2002, p.18). Logrando la retroalimentación asertiva para los actores del proceso de comunicación. Así mismo, "el diálogo, entre docente y el alumno, es condición básica para un aprendizaje practico reflexivo; además, que el maestro trasmite mensajes a sus aprendices tanto en forma verbal como en la forma de ejecutar" (Schön, 1992, p.10). Es decir, a través de su propio modelado.

Como se observa en la tabla 4, el procesamiento arrojó para los docentes un promedio de 3,97 y una desviación de 0,27 , indicando una categoría alta con una muy baja dispersión de las respuestas, al considerar que con alta frecuencia consideran en la planificación de los procesos instruccionales, las características biopsicosociales de los estudiantes, el tiempo, ambiente natural y social, así como el tamaño del grupo.

Es así que, manteniendo la tendencia de las respuestas, los estudiantes perciben que solo de manera moderada los docentes consideran estos aspectos durante la planificación de su estrategia instruccional al arrojar un promedio de 3,04 y, una desviación de 0,43.

Tabla 4. Dimensión: Criterios para la aplicación de las estrategias instruccionales

\begin{tabular}{|l|c|c|c|c|}
\hline \multirow{2}{*}{\multicolumn{1}{|c|}{ Indicadores }} & \multicolumn{2}{c|}{ Docentes } & \multicolumn{2}{c|}{ Estudiantes } \\
\cline { 2 - 5 } & Promedio & Desviación & Promedio & Desviación \\
\hline Características biopsicosociales & 3,94 & 0,27 & 3,04 & 0,40 \\
\hline El tiempo, el ambiente natural y social & 3,97 & 0,30 & 3,04 & 0,44 \\
\hline El tamaño del grupo & 4,00 & 0,23 & 3,04 & 0,42 \\
\hline Promedio de la variable & 3,97 & 0,27 & 3,04 & 0,42 \\
\hline
\end{tabular}

Fuente: Datos obtenidos mediante la aplicación del cuestionario a docentes y estudiantes de las instituciones analizadas (Jiménez, Cujía, Mejía, 2015).

Para Rodrigo \& Arnay (1997), la instrucción es una interpretación, una construcción mental en donde resulta imposible aislar el participante; por esto el aprendizaje es una construcción interior y subjetiva, donde las características biopsicosociales las utiliza el docente como herramientas de aprendizaje, además de los aspectos físicos y sociales que conforman el hecho educativo.

En ese marco de ideas, en relación al primer indicador de esta dimensión, asociando a las características biopsicosociales, el promedio alcanzó para los profesores un valor de 3,94 y una desviación de 0,37, indicando una categoría alta con una muy baja dispersión de las respuestas; se expresa que con esa frecuencia, al realizar una sesión de aprendizaje, toman en cuenta el grado de desarrollo del estudiante como base para el diseño de la estrategia instruccional, evaluando las operaciones cognoscitivas que el educando es capaz de realizar, de manera que contribuye con su aprendizaje y vinculan el conocimiento previo del alumno con la nueva información, y de esta manera se logra un aprendizaje significativo.

Con respecto a los estudiantes el promedio fue de 3,04 y la desviación de 0,40, ubicándose en la categoría moderada con una muy baja dispersión de las respuestas; es decir, una alta confiabilidad de las mismas al coincidir que con esa frecuencia los docentes toman en consideración las características biosicosociales de los alumnos, a la hora de planificar un proceso instruccional.

Por otra parte, APEP (Asociación de Promoción de la Educación Popular, 2009, p.4-5), en el marco de los criterios para la elección de estrategias pedagógicas establece, las características biopsicosociales de los estudiantes, entre ellas su grado de desarrollo, las operaciones cognoscitivas que 
es capaz de realizar, los conocimientos previos, sus motivaciones, permiten al profesor orientar su comportamiento no solo por los objetivos que le plantean sino también por otros que se propone conscientemente, logrando un papel más activo de su conducta.

En cuanto al indicador el tiempo el ambiente natural y social, el procesamiento arrojó para los profesores un promedio de 3,97 y una desviación de 0,30 , ubicándose en la categoría alta con una muy baja dispersión de las respuestas; expresando que con esa frecuencia adecuan su estrategia instruccional en consideración al tiempo del cual dispone para realizar la sesión de aprendizaje y manejan de forma integral los elementos que conforman el hecho educativo, para que los estudiantes aprovechen la experiencia de aprendizaje. Opinaron también que el manejo del tiempo les permite generar programas que contribuyan a optimar el uso de este factor de manera racional.

Con relación a los estudiantes el promedio fue de 3,04 y la desviación de 0,44, indicando una tendencia similar a las dimensiones e indicadores anteriormente analizados, expresando en este sentido que con moderada frecuencia el docente utiliza el tiempo y el ambiente natural y social como criterios para la aplicación de estrategias instruccionales.

La APEP (2009), considera el tiempo previsto para esta actividad en la selección del método o técnica a aplicar y el mismo modo la disponibilidad y características del ambiente, bien sea en las aulas, laboratorio, talleres, biblioteca y otros. El hecho educativo requiere del manejo integral de un conjunto de elementos que deben ser articulados de manera coherente, para lograr procesos de aprendizaje que conduzcan a los cambios esperados en los estudiantes.

En relación al último indicador, referido al tamaño del grupo, como se observa en la tabla 4, el procesamiento de las respuestas arrojó para los docentes un promedio de 4 , y una desviación de 0,23 , indicando una categoría alta con una muy baja dispersión de las respuestas. En este sentido, los profesores manifestaron que con alta frecuencia toman en cuenta el número de alumnos para la aplicación de determinadas técnicas de aprendizaje.

Con respecto a las respuestas de los estudiantes el procesamiento arrojó un promedio de 3,04 y una desviación de 0,42 , indicando una categoría moderada al expresar que solo con esa frecuencia los profesores consideran el tamaño del grupo a la hora de planificar y ejecutar la estrategia instruccional.

Para Díaz \& Hernández (2002), se debe tener presente el número de alumnos para la aplicación de determinadas técnicas según estén dirigidas a grupos en su totalidad o se trate de algunas de las técnicas grupales, en cuyo caso es necesario los alumnos en pequeños grupos. Esto es vital si se pretende alcanzar resultados satisfactorios que conduzcan a un aprendizaje significativo.

Con relación a la variable proceso metacognitivo, el procesamiento de los datos arrojó un promedio para los docentes de 2,99 y una desviación de 0,38 , ubicándose en la categoría moderada con una muy baja dispersión de las respuestas, al expresar que con ese nivel observan el desarrollo de los componentes del proceso metacognitivo en los estudiantes (Tabla 5).

Tabla 5. Variable: Proceso Metacognitivo

\begin{tabular}{|l|c|c|c|c|}
\hline \multirow{2}{*}{\multicolumn{2}{|c|}{ Dimensiones }} & \multicolumn{2}{c|}{ Docentes } & \multicolumn{2}{c|}{ Estudiantes } \\
\cline { 2 - 5 } & Promedio & Desviación & Promedio & Desviación \\
\hline Componentes del proceso metacognitivo & 2,99 & 0,38 & 4,00 & 0,31 \\
\hline Promedio de la variable & 2,99 & 0,38 & 4,00 & 0,31 \\
\hline
\end{tabular}

Fuente: Datos obtenidos mediante la aplicación del cuestionario a docentes y estudiantes de las instituciones analizadas (Jiménez, Cujía, Mejía, 2015). 
Igualmente, con respecto a las respuestas de los estudiantes, el procesamiento arrojó un promedio de 4 y una desviación estándar de 0,42 , indicando una categoría alta con una muy baja dispersión pues, contrariamente a los que expresan los docentes, los alumnos consideran presentar un alto desarrollo de los mencionados componentes.

Para Ann Brown (citado por Bara, 2001, p.68), quien define el proceso metacognitivo en el estudiante como:

El control efectuado de una forma deliberada y consciente de la propia actividad cognitiva. Partiendo de esta propuesta podemos afirmar que las actividades metacognitivas suponen mecanismos de autorregulación y de control que le sirven al sujeto cuando se muestra activo en la resolución de problemas. (Bara, 2001, p.68)

Lo que da a los resultados alcanzados y materializados en un aprendizaje significativo.
Como se observa en la tabla 6 , la dimensión componentes del proceso metacognitivo se ubicó en un nivel moderado para los docentes al arrojar el procesamiento un promedio de 2,99 y, alto para los estudiantes, pues el promedio como anteriormente se mencionó arrojó un promedio de 4 . En este marco de ideas, estos resultados son producto del siguiente análisis por indicador:

En cuanto al indicador conocimiento metacognitivo el procesamiento de los datos arrojó para los docentes un promedio de 2,99 y una desviación de 0,44 , ubicándose en la categoría moderada con una muy baja dispersión de las respuestas. Expresó que con esa frecuencia los estudiantes demuestran capacidad para percibir el conocimiento en cuanto a sus propias competencias en la realización de diversas tareas cognitivas, comprender la influencia de la naturaleza de las tareas que se le asignan sobre la facilidad o dificultad para llevarla a cabo, así como certidumbre en cuanto al uso de diferentes estrategias para desarrollar exitosamente una tarea.

Tabla 6. Dimensión: Componentes del proceso metacognitivo

\begin{tabular}{|l|c|c|c|c|}
\hline \multirow{2}{*}{\multicolumn{1}{|c|}{ Indicadores }} & \multicolumn{2}{c|}{ Docentes } & \multicolumn{2}{c|}{ Estudiantes } \\
\cline { 2 - 5 } & Promedio & Desviación & Promedio & Desviación \\
\hline Conocimiento metacognitivo & 2,99 & 0,44 & 3,97 & 0,36 \\
\hline Regulación de los procesos cognitivos & 2,99 & 0,32 & 4,04 & 0,26 \\
\hline Promedio de la variable & 2,99 & 0,38 & 4,00 & 0,31 \\
\hline
\end{tabular}

Fuente: Datos obtenidos mediante la aplicación del cuestionario a docentes y estudiantes de las instituciones analizadas. (Jiménez, Cujía, Mejía, 2015)

A este tenor los estudiantes ubican sus respuestas en un nivel más alto, pues el promedio alcanzó un valor de 3,97 y una desviación de 0,36; al expresar una alta frecuencia, demuestran las capacidades y competencias anteriormente mencionadas.

En cuanto al último indicador, referido a la regulación de los procesos cognitivos, el procesamiento arrojó para los docentes un promedio de 2,99 y una desviación de 0,32, indicando una categoría moderada con una muy baja dispersión de las respuestas al expresar los profesores que con moderada frecuencia los estudiantes demuestra capacidad para planificar las actividades que realiza durante el proceso de aprendizaje. Según Argüelles (2007), la normalización de los procesos cognitivos hace alusión a la organización, el control y la valoración; se colocan en funcionamiento a fin de garantizar el cumplimiento de los propósitos establecidos de la gestión cognitiva, de tal manera que se desarrollen una educación con un grado mayor de importancia. De ahí la fiscalización y el control que se debe mantener en la operación cognitiva, verificando continuamente el progreso realizado con el propósito de encaminar el trabajo a la realización de las metas propuestas. 
Manifestaron además que con esa moderada frecuencia los estudiantes demuestran capacidad para alcanzar las metas planteadas, y claramente reconocer las dificultades causadas durante el desarrollo de las actividades con la intensión de crear procesos diferenciadores. Por su parte, los estudiantes ubican sus respuestas en un nivel más alto, lo cual indica opiniones diferentes con relación a este indicador, pues consideran que con una alta frecuencia, soportada con un promedio de 4,04 y una desviación de 0,26, demuestran cada una de las competencias necesarias para regular sus procesos cognitivos.

\section{CONCLUSIONES}

Las diferencias en las respuestas entre docentes y estudiantes, con relación a las estrategias instruccionales y la percepción en cuanto al proceso metacognitivo de los alumnos, permitió observar un nivel de correspondencia moderado entre ambas variables; las estrategias aplicadas por los docentes no han permitido consolidar de manera plena los conocimientos y elementos regulatorios que contribuyan al estudiante al manejo efectivo de sus procesos cognitivos y, por consiguiente, a alcanzar una aprendizaje significativo por lo que se sugieren planes de mejoramiento al proceso de gestión.

Al caracterizar los diferentes tipos de estrategias aplicadas por los docentes se observan diferencias significativas entre docentes y estudiantes, lo cual refleja deficiencias en cuanto a las actividades y recursos que los profesores utilizan antes, durante y posterior al desarrollo de la sesión de aprendizaje. Se proponen interacciones entre docente - alumno altamente participativas.

En relación con la dimensión concerniente a los métodos de formación de estrategias instruccionales, se observó también diferencias en las respuestas de docentes y estudiantes. Esto refiere deficiencias importantes con relación al esfuerzo realizado por los profesores dentro del ejercicio de su praxis instruccional. Al tratar de describir los métodos se observó, por un lado una alta aplicación de la información y el modelado por parte de los docentes como mecanismo de apoyo en el proceso de aprendizaje. Y por otro lado se observó que los estudiantes perciben una moderada utilización de estos métodos. Se aconseja estimular la participación del estudiante.

Al tratar de identificar los criterios utilizados por los docentes se observa una tendencia similar en las respuestas de ambas fuentes de información, indicando que el esfuerzo realizado por los docentes no está siendo percibido de manera plena por los estudiantes. Al respecto, para los profesores las características biopsicosociales de los estudiantes, el tiempo, ambiente natural y social, así como el tamaño del grupo, son elementos fundamentales considerados en el diseño y ejecución de su praxis instruccional. Por el contrario, los estudiantes perciben de solo con moderada frecuencia los profesores consideran la aplicación de estos criterios.

Al determinar los componentes del proceso metacognitivo se observó deficiencias importantes con relación a los mismos; aunque lo estudiantes perciben un alto nivel de desarrollo de sus conocimientos y procesos regulatorios necesarios para el manejo de sus aspectos cognitivos, los profesores manifiestan que solo de forma moderada los estudiantes demuestran estas competencias, fundamentales para lograr un aprendizaje significativo.

\section{REFERENCIAS BIBLIOGRÁFICAS}

Agudelo, C. A., Martínez, L. M., \& Ortiz, I. C. (2013). Gestión del Conocimiento: Un activo intangible a través de la investigación. Praxis, 9, 108-113.

APEP. (2009). Manual de Estrategias Metodológicas y Medios Didácticos para el Proceso de Enseñanza y Aprendizaje en el Taller Escolar APEP. Caracas.

Argüelles, D. (2007). Estrategias para promover procesos de aprendizaje autónomo. Bogotá: Alfaomega Colombiana S.A.

Bara, P. (2001). Estrategias metacognitivas y de aprendizaje: estudio empírico sobre el efecto de la aplicación de un programa metacognitivo, y el dominio de las estrategias de aprendizaje en estudiantes de E.S.O., B.U.P. y Universidad. Universidad Complutense de Madrid. Recuperado de http://biblioteca.ucm. es/tesis/edu/ucm-t25562.pdf 
Brown, A. L. (1978). Knowing when, where and how to remember. A problem of metacognition. In. R. Glaser (Ed.), Advances in instructional psychology (I). Hillsdale, N. J.: Erlbaum.

Bunge, M. (1981). La investigación científica. Ariel, Barcelona: Ariel.

Chávez, N. (2007). Introducción a la Investigación Educativa. México: Graficcpress.

Díaz, B. F., \& Hernández R. G. (2002). Estrategias docentes para un aprendizaje significativo. Una interpretación constructivista. México: McGraw Hill.

De la Hera, C. (2004). La mediación como estrategia para la resolución de conflictos: una perspectiva psicosocial. Departamento de Ciencias Sociales. España: Editorial de la Universidad Rey Juan Carlos.

Hernández, R., Fernández, C., \& Baptista, P. (2010). Metodología de la Investigación. México: Mc Graw-Hill.

Hurtado de Barrera, J. (2000). Metodología de Investigación Holística ( ${ }^{\mathrm{a}}$. Ed.). Caracas: Venezuela: Sypal.

Leal, J. (2009). La Autonomía del Sujeto Investigador y la Metodología de Investigación. 2da. Edición.

Maussa, D. E. (2014). Características, principios y fines de la evaluación del desempeño docente. Praxis, 10, 8-20.
Rodrigo, López, M. J., \& Arnay, J. (1997). La construcción del conocimiento escolar. Barcelona: Paidós.

Schön, Donald. (1992). La formación de profesionales reflexivos: Hacia un nuevo diseño de la enseñanza y el aprendizaje en las profesiones. Barcelona: Paidós.

Tamayo \& Tamayo, M. (2007). El proceso de la investigación científica. México: Limusa.

Tobón, S. (2010). Formación basada en competencias. México: Ecoe.

Valencia, L., Gil, J., Betancurt. F., Giraldo. C., \& Orozco. S. (2011). Implementación de estrategias pedagógicas innovadoras para el mejoramiento del nivel de desempeño académico y reducción del déficit de atención en los estudiantes de tercer grado de primaria del colegio cooperativo de apartado. Editores Universidad Católica de Manizales. Facultad de Educación. Medellín. Colombia.

Vieytes, R. (2004). Metodología de la Investigación en Organizaciones, Mercado y Sociedad. Epistemología y Técnicas. 1era Edición. Buenos Aires: Editorial de las Ciencias. 